\title{
The role of neuromuscular blocking drugs in early severe acute respiratory distress syndrome
}

\author{
Craig J. Needham, MD • Peter G. Brindley, MD
}

Received: 8 August 2011/Accepted: 18 October 2011/Published online: 1 November 2011

(C) Canadian Anesthesiologists' Society 2011

\begin{abstract}
Background Acute respiratory distress syndrome (ARDS) is defined as severe hypoxemic respiratory failure resulting from diffuse lung injury and secondary to direct and indirect insults. Despite advances, mortality remains as high as 40-60\%. Neuromuscular blocking agents (NMBAs) are used to facilitate mechanical ventilation in patients with ARDS and have been shown to improve arterial partial pressure of oxygen. However, the association between NMBAs and mortality is unclear. Furthermore, morbidity concerns exist, particularly regarding a putative role in intensive care unit (ICU)acquired weakness.

Objective The purpose of this study was to compare survival in adult patients with early ARDS who were randomized to receive either a 48-hr infusion of the NMBA, cisatracurium, or a placebo.
\end{abstract}

Author contributions Craig J. Needham made significant contributions to the conception, design, and writing of this article. Peter G. Brindley contributed to both the article's draft and its critical revision for important intellectual content. He also gave final approval to the version to be published.

Article appraised Papazian L, Forel JM, Gacouin A, et al. Neuromuscular blockers in early acute respiratory distress syndrome. N Engl J Med 2010; 363: 1107-16.

\section{J. Needham, MD ( $\square)$}

Department of Anesthesiology and Pain Medicine,

University of Alberta, Room CSB 8-120,

Edmonton, AB T6G 2B7, Canada

e-mail: needham@ualberta.ca

P. G. Brindley, MD

Division of Critical Care Medicine,

University of Alberta, Edmonton,

$\mathrm{AB}$, Canada
Design and setting This study was a multicentre doubleblinded randomized controlled trial involving 20 ICUs in France from March 2006 to March 2008.

Patients Eligible patients were $>18 y \mathrm{r}$ with an intubated trachea and ventilated lungs for acute hypoxemic respiratory failure. Their $\mathrm{PaO}_{2} / \mathrm{F}_{1} \mathrm{O}_{2}$ ratio was $<150$ at a tidal volume of $6-8 \mathrm{~mL} \cdot \mathrm{kg}^{-1}$ ideal body weight and a positive end-expiratory pressure $(\mathrm{PEEP}) \geq 5 \mathrm{~cm} \mathrm{H}_{2} \mathrm{O}$ for $<48 \mathrm{hr}$. Additional inclusion criteria were radiographic evidence of bilateral pulmonary infiltrates and the absence of left atrial hypertension. Exclusion criteria included patients already receiving NMBA at enrolment; those who had increased intracranial pressure, severe chronic respiratory disease, or severe chronic liver disease; those who had received a bone marrow transplant or had chemotherapy-induced neutropenia; those who had a pneumothorax; and those who were expected to require mechanical ventilation for $<48 \mathrm{hr}$ or were enrolled in another trial within 30 days.

Intervention Three hundred twenty-six patients were screened, and 340 of these underwent randomization in blocks of four and received either a 48-hr infusion of cisatracurium (15 mg bolus followed by $37.5 \mathrm{mg} \cdot \mathrm{hr}^{-1}$ ) or a volume equivalent placebo. One hundred and seventy-eight patients received a cisatracurium infusion, and one patient withdrew leaving 177 patients included in the analysis. One hundred and sixty-two patients received the placebo infusion. Prior to either infusion, patients were sedated to a Ramsay sedation score of 6 . Patients' lungs were ventilated by a volume assist-controlled mode according to the ARDS Clinical Network Mechanical Ventilation Protocol (http:// www.ardsnet.org/) with the goal $\mathrm{SpO}_{2}$ of $88-95 \%$ (or $\mathrm{PaO}_{2}$ $55-80 \mathrm{mmHg}$ ) and goal plateau pressure $\leq 35 \mathrm{~cm} \mathrm{H}_{2} \mathrm{O}$. Open-label boluses of cisatracurium $20 \mathrm{mg}$ (maximum of two per 24-hr period) were allowed if plateau pressures 
remained $>32 \mathrm{~cm} \mathrm{H}_{2} \mathrm{O}$ despite increased sedation and despite decreased PEEP and decreased tidal volumes. Monitoring of paralysis via peripheral nerve stimulation was not permitted.

Measurements The primary outcome was death before hospital discharge and within 90 days of study enrolment. It was determined a priori that this would be adjusted for imbalance in key risk factors at baseline, as derived from Cox regression. Secondary outcomes included 28-day mortality, number of ventilator-free days, number of days outside of ICU, number of days without organ system failure, rate of barotrauma, and rate of ICU-acquired paresis (as defined by a Medical Research Council [MRC] score < 48) on day 28 and at ICU discharge.

Main results With regard to the primary outcome, crude 90-day mortality was $31.6 \%$ in the cisatracurium group vs $40.7 \%$ in the placebo group. This outcome did not reach statistical significance $(P=0.08)$. However, post hoc analysis found a reduction in 90-day mortality in the cisatracurium group compared with placebo $195 \%$ confidence interval 0.48 to $0.98 ; P=0.04$ ). Results suggest that the reduction in 90-day mortality in the cisatracurium group was confined to those patients with a $\mathrm{PaO}_{2} / \mathrm{F}_{1} \mathrm{O}_{2}$ ratio $<120$. Additionally, 28-day mortality was significantly lower in the cisatracurium group (absolute difference $-9.6 \% ; P=0.05$ ). The cisatracurium group also had significantly more ventilator-free days, more days outside of the ICU, and more days free of organ-failure. Similarly, pneumothorax developed more often and earlier in the placebo group than in the cisatracurium group. The rate of ICU-acquired weakness at day 28 or at ICU discharge did not differ significantly between the two groups. Conclusions Treatment in early severe ARDS with the NMBA, cisatracurium, for $48 \mathrm{hr}$ was associated with lower adjusted 90-day mortality. It was also associated with decreased morbidity, which included increased ventilatorfree days, increased ICU-free days, and increased organ failure-free days. These benefits occurred without increasing the incidence of ICU-acquired weakness.

\section{Commentary}

Current state of the literature and study relevance

First described in the $1960 \mathrm{~s}$, acute respiratory distress syndrome is defined by lung injury causing hypoxemia refractory to oxygen therapy along with bilateral lung infiltrates and non-cardiogenic pulmonary edema. ${ }^{1}$ It is also associated with decreased lung compliance, a mortality of $\geq 40 \%$, and substantial morbidity even years on. ${ }^{2}$ Extensive research has concluded that a variety of non- ventilatory strategies, such as nitric oxide, prone position, and corticosteroids, can improve clinical parameters, but they are not clearly associated with a survival benefit. ${ }^{3}$ In fact, it has become increasingly apparent in the literature that optimizing gas exchange (i.e., normalizing partial pressures of oxygen or carbon dioxide) is not clearly associated with improved clinical outcome. As such, the focus has shifted to how best to "protect" the lung, which has become synonymous with minimizing ventilatorinduced lung injury and mitigating the progression to multisystem organ failure. ${ }^{4}$

Lung protective strategies employ low tidal volumes. However, this approach can result in air hunger and increased respiratory drive. In order to minimize patientventilator dysynchrony, patients require adequate sedation, and surveys reveal that $25-55 \%$ of ARDS patients receive adjuvant NMBAs. ${ }^{5}$ This is important because NMBAs are not believed to be risk-free. For example, critical illness polyneuropathy, critical illness myopathy, and acute myopathic syndromes have all been associated with prolonged neuromuscular blockade. Furthermore, ICU-acquired weakness is associated with increased ICU stay, increased hospital stay, and also increased mortality. ${ }^{6-8}$ Therefore, guidelines regarding the use of NMBAs in the ICU typically caution that they should be limited to refractory hypoxemia and hypercarbia, and they should be short-term and guided by train-of-four monitoring. ${ }^{9}$

Despite suggestions that NMBAs negatively affect both morbidity and mortality in critically ill patients, there has been a paucity of confirmatory data from randomized trials. In contrast, Gainnier et al. showed that a 48-hr infusion of cisatracurium in ARDS was associated with an increase in arterial partial pressure of oxygen (as compared with placebo), and this increase was sustained for $120 \mathrm{hr}$. However, their study included only four centres and only 56 patients. Also, it was not powered adequately for mortality, though the authors did report a non-significant trend toward decreased ICU stay and decreased 28-day mortality (favouring cisatracurium). ${ }^{10}$ In a follow-up study, Forel et al. used a similar study design and found that early use of cisatracurium infusion in ARDS also decreased serum and pulmonary concentrations of pro-inflammatory markers when compared with control. ${ }^{11}$ The current work by Papazian et al. has built on these studies. This larger 20-centre trial, dubbed ACURASYS, was designed and executed in hopes of better elucidating the risks $v s$ the benefits of NMBAs in adult patients undergoing lungprotective ventilation for severe ARDS.

Analysis of methodology

The authors should be commended for their considerable efforts to minimize bias. First, the ACURASYS study was 
prospective randomized placebo-controlled and doubleblinded. Additionally, it was multicentred, and both medical and surgical patients were included, thereby incorporating a variety of causes for ARDS. Important pharmacologic and non-pharmacologic interventions were tracked meticulously, and aside from the use of open-label cisatracurium (discussed below), the interventions did not differ significantly between the study-drug and placebo groups. Patients were block randomized in a stratification scheme designed to control for study centre, age ( $\leq 60 \mathrm{yr}$ or $>60 \mathrm{yr}$ ), and duration of ventilation at baseline ( $\leq 48 \mathrm{hr}$ or $>48 \mathrm{hr}$ ). Data were analyzed on an intent-to-treat basis, and the investigators achieved complete follow-up. Data collection both before and after randomization focused on key demographic, physiologic, and interventional factors.

Most clinically relevant parameters were captured. However, detractors have criticized the fact that dysynchrony and spontaneous respiratory efforts were not monitored formally, as this might have predisposed to worse outcomes in the placebo group. To reduce dysynchrony (which would also have jeopardized blinding), both groups were sedated to a Ramsay sedation score of 6 (as defined by "no response to glabellar tap"). In addition, they allowed 20-mg open-label cisatracurium boluses for excessive plateau pressures (which is arguably necessary for patient safety and study equipoise). However, this approach has the potential to confound analysis. Indeed, twice as many patients in the placebo group required cisatracurium boluses in the first $48 \mathrm{hr}$. Given that the bolus dose was small in comparison with the infusion, the total dose of NMBA remained considerably higher in the cisatracurium group. As such, the study and placebo groups can still be compared meaningfully. Additionally, this aspect of the study likely reflects "real world" ICU practice.

Detractors will also highlight, justifiably, that the primary outcome was adjusted (i.e., the crude 90-day mortality did not differ significantly, but there was a significant difference in the adjusted mortality). Despite stating a priori that the primary outcome would be adjusted based on imbalances identified between groups, this approach inherently weakens the validity of the results. Indeed, this is likely a chief source of concern when interpreting the authors' conclusions because the observed "treatment effect" on mortality was not observed between the originally allocated groups but rather after correction for differences between groups. It is problematic to evaluate whether a treatment effect is due to chance when the process of randomization is ignored in the final analysis. Also, the protocol allowed for a wide range of positive endexpiratory pressure (PEEP) levels. This might confound data given that higher PEEP might improve oxygenation and reduce ventilator-induced lung injury but also cause circulatory depression and lung injury from excess distension. However, the supplementary appendix suggests that PEEP did not differ between groups at baseline, $24 \mathrm{hr}$, or $72 \mathrm{hr}$.

Additionally, the study was underpowered. It was originally intended to detect a $15 \%$ difference in mortality, which was based on an assumed control mortality of $50 \%$. In contrast, the placebo mortality was almost $10 \%$ lower (40.7\%), and as a result, 885 additional patients would have been required. Adequate power reduces type II error (i.e., the likelihood of missing a treatment effect when one does truly exist). Although a treatment effect was seen in this study, its conclusions must be evaluated in light of the fact that it was not powered adequately to detect said effect.

Relevance to clinical practice

(strengths/weaknesses/external validity)

Despite clinically provocative results, questions remain as to the generalizability of this study. For example, only a single benzylisoquinoline NMBA was used (cisatracurium), whereas steroidal agents (i.e., vecuronium, rocuronium, pancuronium) might lead to higher incidences of myopathy..$^{12}$ Moreover, NMBAs were used only early in ARDS and for only $48 \mathrm{hr}$ - a period that may be too brief for ICUparesis. Post hoc analysis established that benefits were seen only in the two-thirds of patients with very severe disease $(\mathrm{PaO} 2 / \mathrm{FiO} 2[\mathrm{P} / \mathrm{F}]$ ratio $<120)$. However, clinicians may have no option but to use NMBAs in such sick patients. If so, then ACURASYS may offer clinicians comfort regarding the use of NMBAs (at least in the short term) rather than increasing NMBA usage in those with better P/F ratios. Questions remain regarding other NMBAs for longer-term usage or for patients with less severe disease. It is also unclear why the Kaplan Meier survival curves did not separate until day 18 despite $48 \mathrm{hr}$ of NMBA administration.

ACURASYS raises the issue of NMBA infusions when ICUs typically use intermittent boluses and as directed by peripheral nerve stimulators. The relatively high initial dose as well as a high infusion rate $\left(>0.2 \mathrm{mg} \cdot \mathrm{kg}^{-1}\right.$ and $5 \mu \mathrm{g} \cdot \mathrm{kg}^{-1} \cdot \mathrm{min}^{-1}$ for a $70-\mathrm{kg}$ patient) are also noteworthy. Also, the dose was not adjusted by patient weight. However, the rationale was to ensure complete blockade and avoid the need for neuromuscular monitoring (which would have impaired blinding). Furthermore, it makes the results more noteworthy given that there was no statistically significant increase in weakness at ICU discharge or at day 28 despite comparatively high doses of NMBA.

\section{Clinical perspective}

Prior to ACURASYS, many clinicians might have freely accepted that NMBAs are associated with increased ICU 
mortality, more weakness, prolonged ventilation, and lengthier ICU stay. As such, it is important that this large prospective study of paralytic agents in ARDS has shown not only a possible benefit (in the most severe cases) but also no obvious downside (in the short term). In brief, this study is clinically significant regardless whether the data are always statistically significant. The authors admit that future studies are warranted before they expect widespread clinical change. As such, it is difficult to offer prescriptive advice regarding NMBA use. However, ACURASYS does justify short-term NMBA usage in severe ARDS.

Also, ACURASYS should stimulate clinical debate. For example, the discussion may change substantially from establishing why NMBAs were considered detrimental to establishing how they might be beneficial. As stated by the authors, this is "speculative". This uncertainty is not surprising, as clinicians still debate the relative importance of volutrauma $v s$ barotrauma $v s$ atelectotrauma $v s$ biotrauma. ${ }^{4}$ In other words, while we have physiologic uncertainty, we will also have clinical uncertainty. The benefits of NMBAs might result from less asynchrony-related end-inspiratory overdistension or from less end-expiratory alveolar collapse. The benefit of NMBAs may also be associated with decreased inflammation (lung or systemic) that might otherwise amplify multisystem organ failure. However, to make informed clinical decisions, we really need to know which inflammatory mediators are beneficial and which are deleterious. Profound sedation, but without NMBAs, might also produce similar improvements. Intensive care unitassociated weakness may even be a form of "organ failure" associated with illness of higher severity and therefore unrelated to the use of NMBAs. Regardless, clinical practice appears to be evolving from an effort to normalize physiology to an effort to preserve organ function and protect our patients from harm. Optimization of ventilation facilitated by the use NMBAs in the critical care setting has an integral role in certain clinical settings.
Funding sources N/A

Conflicts of interest None declared.

\section{References}

1. Ware LB, Matthay MA. The acute respiratory distress syndrome. N Engl J Med 2000; 342: 1334-49.

2. Herridge MS, Tansey CM, Matte A, et al. Functional disability 5 years after acute respiratory distress syndrome. N Engl J Med 2011; 364: 1293-304.

3. Alsaghir AH, Martin CM. Effect of prone positioning in patients with acute respiratory distress syndrome: a meta-analysis. Crit Care Med 2008; 36: 603-9.

4. Slutsky AS. Neuromuscular blocking agents in ARDS. N Engl J Med 2010; 363: 1176-80.

5. Raoof S, Goulet K, Esan A, Hess DR, Sessler CN. Severe hypoxemic respiratory failure: part 2-nonventilatory strategies. Chest 2010; 137: 1437-48.

6. Vender J, Szokol J, Murphy GS, Nitsun M. Sedation, analgesia and neuromuscular blockade in sepsis: an evidence-based review. Crit Care Med 2004; 32: S554-61.

7. Deem S, Lee CM, Curtis JR. Acquired neuromuscular disorders in the intensive care unit. Am J Respir Crit Care Med 2003; 168: 735-9.

8. Griffiths RD, Hall JB. Intensive care unit-acquired weakness. Crit Care Med 2010; 38: 779-87.

9. Murray MJ, Cowen J, DeBlock H, et al. Clinical practice guidelines for sustained neuromuscular blockade in the acult critically ill patient. Crit Care Med 2002; 30: 142-56.

10. Gainnier M, Roch A, Forel JM, et al. Effect of neuromuscular blocking agents on gas exchange in patients presenting with acute respiratory distress syndrome. Crit Care Med 2004; 32: 113-9.

11. Forel JM, Roch A, Marin V, et al. Neuromuscular blocking agents decrease inflammatory response in patients presenting with acute respiratory distress syndrome. Crit Care Med 2006; 34: 2749-57.

12. Testelmans $D$, Maes $K$, Wouters $P$, et al. Rocuronium exacerbates mechanical ventilation-induced diaphragm dysfunction in rats. Crit Care Med 2006; 34: 3018-23. 